\title{
The fungal nature of Pneumocystis
}

\author{
J. R. STRINGER, ${ }^{1}$ J. C. EDMAN, ${ }^{2}$ M. T. CUSHION ${ }^{3}$, F. F. RICHARDS ${ }^{4}$ AND \\ J. WATANABE \\ ${ }^{1}$ Department of Molecular Genetics, Biochemistry and Microbiology, University of \\ Cincinnati, Cincinnati, Ohio, ${ }^{2}$ Hormone Research Institute, University of California, \\ San Francisco, ${ }^{3}$ University of Cincinnati/VA Medical Center, Cincinnati, Ohio, and \\ ${ }^{4}$ Yale University, New Haven, USA; ${ }^{5}$ Institute of Medical Science, University of \\ Tokyo, Tokyo, Japan
}

Pneumocystis carinii is an opportunistic protistan pathogen that causes a lethal pneumonia in immunocompromised patients. The organism was described in the early 1900s by Chagas, who suggested that it was a trypanosome [6]. After World War II, $P$. carinii was recognized as an important cause of morbidity and mortality in malnourished children. With the advent of organ transplantation and the attendant use of immunosuppressive agents, the pathogen again emerged as a significant problem. Drugs were developed to treat $P$. carinii pneumonia in the $1970 \mathrm{~s}$, but these agents have proved to be less effective in managing the disease in patients with AIDS, and most eventually perish from a $P$.carinii infection [33].

Although $P$. carinii is now a major medical problem, little is known about the organism and its normal and pathological interactions with mammalian hosts. $P$. carinii has been refractory to cultivation and this has restricted the application of biochemical and genetic techniques. In the absence of solid biochemical and genetic data, many basic questions remained unanswered. One such question was the delineation of the proper phylogenetic associations of $P$. carinii. Prior to 1988 , classification of $P$. carinii was based primarily on morphology, ultrastructure, growth requirements and susceptibility or resistance to chemotherapeutic agents. Based on these fairly weak criteria, $P$. carinii was widely considered to be a protozoan, although some investigators had argued that $P$. carinii was more similar to the fungi than to the protozoa [31]. The first molecular genetic evidence bearing on the taxonomy of $P$. carinii was at odds with the prevailing notion. Three groups found that the sequences of $P$. carinii ribosomal RNAs and ribosomal RNA genes were much more similar to those of fungi than to those of any known protozoan $[16,29,34]$. Over the last 3 years, data have accumulated that support the idea that Pneumocystis is a fungus. However, it is clear that this parasitic fungus is not typical of the fungi most often isolated and studied in either the basic or clinical laboratory.

\section{Molecular genetics}

Data derived from molecular cloning of three other $P$. carinii genes, thymidylate synthase [17], dihydrofolate reductase [15] and a P-type cation-translocation ATPase

Correspondence address: J. R. Stringer, Department of Molecular Genetics, Biochemistry and Microbiology, 3110 Medical Sciences Building, University of Cincinnati, 231 Bethesda Avenue, Cincinnati, Ohio, USA. 
(Stringer et al., Abstract S22.3, 11th Congress of ISHAM, Montreal, Canada, June 24-28, 1991), have supported the idea that $P$. carinii is a fungus.

Both the $P$. carinii thymidylate synthase and dihydrofolate reductase genes were found to encode predicted polypeptide chains that were somewhat more closely related to the corresponding proteins from Saccharomyces cerevisiae than to the corresponding proteins from non-fungi. In addition, the $P$. carinii dihydrofolate reductase deduced amino acid sequence contained 206 residues, which like the $S$. cerevisiae enzyme, is slightly larger than the mammalian and bacterial enzymes. Both $P$. carinii genes contained small introns which are typical of those found in other fungal genes.

In $P$. carinii, the genes for thymidylate synthase and dihydrofolate reductase are not linked, but reside on different chromosomes. This contrasts with the situation in the parasitic protozoa, Leishmania [4] and Plasmodium [5], both of which package these two enzymatic activities in a single peptide encoded by a single gene. The conservation of this bifunctional protein in these two protozoa is striking because Leishmania and Plasmodium are not close relatives. While there is no proof that protozoa cannot encode monofunctional thymidylate synthase and dihydrofolate reductase, the presence of these monofunctional genes in $P$. carinii supports the contention that P.carinii is fungus.

Edman has recently analysed the sequences of thymidylate synthase molecules from diverse eucaryotic species. A phylogenetic tree inferred from this comparison placed $P$. carinii on the fungal branch (Edman, J. C., Abstract S22.1, 11th Congress of ISHAM, Montreal, Canada, June 24-28, 1991).

P-type cation-translocation ATPases are enzymes that serve to pump cations across cellular membranes. The genes that encode P-type cation-translocation ATPases are found in diverse eucaryotes as well as in selected prokaryotes. All P-type ATPases share certain structural characteristics, but fungal proton-pumping ATPases appear to share at least one domain not found in other P-type ATPases. A P-type ATPase gene isolated from $P$. carinii has been found to contain this fungal-protonpump domain (Stringer et al., Abstract S22.3, 11th Congress of ISHAM, Montreal, Canada, June 24-28, 1991). These data are in complete agreement with the idea that $P$. carinii is a fungus.

If $P$. carinii is a fungus, what can be said about its position within that kingdom? The molecular genetic data at this point seem paradoxical. Analysis of the 16s-like rRNA sequence suggested that $P$. carinii may be an ascomycete $[16,29]$, but analysis of 5s rRNA suggested that $P$. carinii was not an ascomycete, but was more similar to the Rhizopoda, Myxomycota and Zygomycota [34]. It is not possible to resolve this paradox at this point. On the one hand, the 16 s-like data are attractive because these RNA species are large enough to include regions that are highly conserved, regions that are highly divergent and regions that are moderately divergent. This range of variation facilitates detection of distant relatives as well as discrimination between very close relatives. Unfortunately, relatively few fungal 16s-like rRNAs have been sequenced and we know of no published data on species from the Rhizopoda, Myxomycota and Zygomycota. By contrast, the 5s rRNA sequence data base is very large, including many fungal species. However, $5 \mathrm{~s}$ rRNAs are quite short and are very highly conserved, and this limits their utility as a phylogenetic index.

Whatever its proper niche within the fungal kingdom, molecular genetic data suggest that there are probably numerous varieties of Pneumocystis in nature. 
P. carinii was originally defined by the histopathology of Pneumocystis pneumonia, and parasites displaying these histopathological characteristics, although from diverse mammalian hosts, are currently all classed under this rubric. Antigenic variability has been observed among Pneumocystis, but the basis for these differences is not clear. Recently, genetic variability among organisms classified as $P$. carinii on the basis of histopathology has been detected by pulsed field electrophoresis. Several laboratories have applied pulsed field electrophoresis to derive electrophoretic karyotypes for Pneumocystis isolated from experimentally infected rats [18, 21, 35]. A common finding was that $P$. carinii populations isolated from different colonies of infected rats each displayed a distinct electrophoretic karyotype. In one study [18], electrophoretic karyotypes were found to be stable for over a year. While each karyotype was different, the number and size range of the chromosomes was usually similar. From the electrophoretic band patterns, the genome of Pneumocystis has been estimated to contain approximately $1 \times 10^{7}$ basepairs. The genome is not comprised entirely of unique sequences. A repeated DNA isolated from rat-derived Pneumocystis was found to compose approximately 7\% of the parasite genome [28]. The repeat was present on each of the $16 P$. carinii chromosomes resolved by field inversion gel electrophoresis. It is not known if this repeated DNA is also present in Pneumocystis from other host species. The basis for karyotype variation in Pneumocystis is not understood. To address this question, DNA probes that hybridize specifically to each chromosome are needed. At present, such markers exist for all but two of the bands seen in a typical Pneumocystis karyotype (Stringer et al., Abstract S22.3, 11th Congress of ISHAM, Montreal, Canada, June 24-28, 1991).

A second line of evidence indicating genetic variation among Pneumocystis was provided by sequence analysis of part of an rRNA gene from rat-derived and humanderived Pneumocystis [32]. While these data are not yet complete enough to support a firm conclusion, they are consistent with the idea that the organisms found in the lungs of rats and humans are probably distinct species.

\section{Morphology, cell structure and cell composition}

The primary site of Pneumocystis infection is the mammalian alveolus, although an increasing number of disseminated cases have been reported in AIDS patients. A myriad of morphological forms exist extracellularly within the mammalian host. The two most numerous morphological forms are the cyst and trophic form. The precyst form has been identified frequently. As a definitive life-cycle has not been identified, terms such as early, intermediate and late will be avoided since they infer a progression or specific life-cycle pathway.

Ultrastructural features of the spherical to ovoid cyst $(5-8 \mu \mathrm{m})$ include a thick cell wall/envelope and up to eight individual intracystic bodies (also known as daughter forms or sporozoites) which may be spores. Each intracystic body has a nucleus, a mitochondrion, free ribosomes and endoplasmic reticulum contained within a double membrane. It is presumed that the released intracystic bodies give rise to the trophic forms. The cyst cell envelope contains a membrane in addition to the plasma membrane as well as carbohydrate, protein and presumably lipid moieties [10]. The presence of the membrane was shown by freeze fracture and transmission electron microscopy as well as by the use of lipid stains [13]. This outer electron dense layer is separated from the intracystic bodies bounded by the plasma membrane, by an 
electron-lucent space. A polar thickening is frequently observed, evident as an enlargement of the middle lucent space. The cyst wall stains with tinctorial methods frequently used to detect fungal cells, including methenamine silver or toluidine blue O. Giemsa staining reveals the nuclei and cytoplasm of the intracystic bodies, but the wall excludes the dye and appears as a halo.

Trophic forms are smaller than cysts $(1-4 \mu \mathrm{m})$ and occur as individual unicellular entities, but more frequently are found in clusters together with the other morphological forms that populate the alveolar lumen. Ultrastructural features include a double nuclear envelope, a nucleolus, glycogen granules, endoplasmic reticulum, ribosomes and one or more mitochondria. Transmission electron microscopy has shown the mitochondrion to contain plate-like cristae [26], a feature typical of fungi. Trophic forms generally appear pleomorphic when viewed after fixation, but observation of unfixed preparations using phase contrast or interference microscopy shows the trophic form to be ellipsoidal in shape without ameboid features. Locomotion has not been directly observed and structures associated with movement (e.g. flagella) have not been detected. Although the trophic form may form a tight interdigitation with the Type I pneumocyte, the precise mechanism of this attachment is as yet unknown. This form is delimited by a double membrane, spanning approximately $20-30 \mathrm{~nm}$. Several investigators have reported membranous vesicles or tubular extensions to arise from the outer membrane [3, 23].

Recent ultrastructural analyses have identified a putative Golgi complex and lysosomes in rabbit-derived Pneumocystis [24]. Golgi were shown to be more numerous in cysts than in the trophic forms.

The cyst wall is susceptible to zymolyase, the key enzyme of which is $\beta-1,3-$ glucan laminaripentoaohydrolase, suggesting the presence of $\beta-1,3$-glucans in the cyst wall. Accordingly, echinocandins and papulocandins, two presumptive $\beta$-1,3-glucan synthesis inhibitors were effective against the parasite in vivo [27]. Indeed, glucose appears to be the predominant monomer in gas chromatography-mass spectroscopy (GC-MS) analyses and the organism has been shown to stain avidly with Concanavalin A (Con A), a lectin which binds mannose and glucose residues.

Fluoresceinated lectins have been used to detect sugars on the surface of cysts $[8,14]$. Organisms derived directly from rat lungs showed strong binding to the lectins Con $\mathrm{A}$, wheat germ agglutinin and soya bean agglutinin indicating the presence of glucose/mannose, $\mathrm{N}$-acetylglucosamine ( $\mathrm{GlcNAc})$ and galactose/ $N$-acetyl galactosamine, respectively. Lectin binding studies were verified by GC-MS analyses of material released from cysts by zymolyase treatment [12]. Glucose was found to be the major constituent, while mannose and galactose were present in equivalent amounts and GlcNAc in lesser quantity. Trace amounts of ribose and sialic acid were detected. In contrast, similar analyses of human derived organisms by high performance liquid chromatography, detected the presence of mannose, glucose, galactose and glucosamine in equimolar concentrations, whereas rat-derived organisms contained higher concentrations of glucose and mannose than other sugars.

Lipid analyses of whole organisms and cell wall material identified palmitate $(16: 0)$ as the most abundant fatty acid [19]. Few differences between the organism lipid profile and that of the host cell controls were observed. Remarkable was the absence of ergosterol and presence of cholesterol as the organism's major sterol. These analyses support previous freeze fracture studies using filipin probes [36]. The absence of ergosterol may explain the resistance of the parasite to amphotericin $B$. 
Proteins are a major constituent of the surface of the organism and their characterization is a focus of research in several laboratories. The most prominent antigen migrates at $116 \mathrm{kDa}$ on sodium dodecyl sulphate-polyacrylamide gel electrophoresis. This protein is thought to be a glycosylated peptide approximately $66 \mathrm{kDa}$ in size.

\section{In vitro cultivation}

Early attempts to grow the aetiological agent of a pneumonia that plagued orphanages during the late 1940s and 1950s resulted in reports of 'yeast-like colonies' on solid agars which resembled Candida and Saccharomyces species [7]. The agent of infection was later identified in histological sections as Pneumocystis. Whether or not these early cultures were actually Pneumocystis has never been confirmed. The exact formulations of these growth media have been lost over time. In the late 1970s the limited growth of rat-derived Pneumocystis on primary cell isolates [25] and established cell lines $[2,20]$ was reported. A number of other cell lines have been reported to also support limited growth $[1,11]$. Although different methods have been used to quantify the organisms, the continuous growth of Pneumocystis has not been achieved.

Two laboratories $[9,30]$ have reported limited replication in axenic culture. Results were as good as those achieved in tissue culture, but subculturing attempts were unsuccessful. It appears that the organism depletes intracellular pools of required growth factors over time in culture and then dies or becomes dormant.

\section{Life-cycle and transmission}

Since a culture system does not exist, the life-cycle remains a matter of conjecture based on interpretation of the static images obtained by ultrastructural and light microscopic techniques. Proposed life-cycles range from simple modes of replication involving binary fission to highly complex branched schemes with asexual and sexual cycles. Observation of synaptonemal complexes in putative precyst stages provided some evidence for a sexual cycle [22].

A major unresolved issue continues to be identification of the agent of Pneumocystis infection. The general human population becomes seropositive to antigens from the organism by the age of 4 years [10]. It is presumed to be disseminated by an airborne particle, based on caging studies performed in the late 1970s and early 1980s. Yet, the question of the origin of infection in the immunocompromised host remains unanswered. Do we carry latent organisms that can reactivate once our immune system breaks down, or do the organisms we are putatively exposed to on a daily basis cause the infection?

Recent studies (Cushion, M., Abstract 522.2, 11th Congress of ISHAM, Montreal, Canada, June 24-28, 1991) used the immunosuppressed rat model of infection combined with electrophoretic karyotyping to show that: (i) heterogeneity exists between organism isolates obtained from different rat strains; (ii) latent organisms acquired while the rat was immunocompetent reactivate upon immunosuppression and cause infection; (iii) infection of a specific Pneumocystis karyotype can be passed from infected rats to naive rats housed within the same room, but not in direct contact.

To execute these studies, viral-antibody-positive rats (indicative of exposure to 
common rodent pathogens and also to Pneumocystis due to open rack housing at the vendor facilities) and viral-antibody-negative rats (barrier raised rats, not exposed to environment/common rodent pathogens, Pneumocystis-free) were either housed under barrier conditions to prevent contact with the environment or in conventional cages which allowed contact with potentially contaminated air. To assess reactivation of latent organisms as a cause of infection, viral-antibody-positive rats were received into facilities in filtered containers and placed immediately under barrier. Immunosuppression began after 7-10 days of acclimatization. After 8-10 weeks of immunosuppression, all rats had Pneumocystis pneumonia. Thus, reactivation of latent organisms can cause infection. Electrophoretic karyotypes obtained from these rats showed patterns specific to each rat strain. Whether this is a function of strain specificity, or infection by an isolate that has established itself within a given room is not yet known.

That an environmental source of Pneumocystis also could cause infection was illustrated by the appearance of infection in naive, immunosuppressed rats placed in a conventional colony of infected rats. The same karyotype of the Pneumocystis harbored within this infected colony was found in the rats introduced into the same room.

Other factors that might be associated with the appearance of Pneumocystis infection including, the presence of anti-Pneumocystis antibodies and antibodies to common rodent pathogens, have been examined (M. T. Cushion et al., unpublished data). The presence or absence of antibodies to the organism did not appear to influence the infection in any way. Rat corona virus was positively associated with the infection in one rat strain, but its absence in other rat strains did not appear to affect the occurrence or severity of infection. However, when an attempt was made to establish a mixed infection by exposure of two different viral-antibody-positive rat strains harboring two distinct Pneumocystis karyotypes, the majority of the rats which were antibody positive to a single virus died as a result of exposure to the other rat strain which was reactive to several viruses. Death was independent of Pneumocystis infection.

\section{Future research challenges}

Progress in $P$. carinii research has accelerated in the last few years, but many questions remain unanswered. We do not know how many species of $P$. carinii exist in humans, or in animals, or whether animals harbor $P$. carinii that can infect humans. Airborne forms of the organism seem to exist, at least in rooms containing colonies of infected rats, but it is not clear whether or not people are exposed to the pathogen under ordinary circumstances. We know little about how $P$. carinii infections are transmitted and established, nor do we know the extent to which $P$. carinii maintains itself in asymptomatic subjects or about the factors needed to convert from asymptomatic to symptomatic infections. Host defenses against $P$. carinii are thought to involve macrophages and T-cells, but the role of other arms of the immune system has not been well studied. Both animal models of $P$. carinii infection and in vitro propagation will need to be improved in order to increase the pace of research into this important pathogen. Having gained the understanding that we are dealing with a fungus should help shape the direction of future investigations. 


\section{CONTRIBUTORS}

The contributors to this symposium were: J. C. Edman, Molecular biological evidence demonstrating the close relationship of $P$. carinii with the fungi; M. Cushion, The cell biology of P. carinii; J. R. Stringer, Analysis of the Pneumocystis genome; F. F. Richards, $P$. carinii infections, some unresolved questions; J. Watanabe, Phylogenetic association of $P$. carinii with the Rhizopoda/Myxomycota/Zygomycota group. The coconvenors were J. R. Stringer and J. C. Edman.

\section{REFERENCES}

1. Armstrong, M. Y. K. \& Richards, F. F. 1989. Propagation and purification of rat Pneumocystis carinii in short term cell culture. Journal of Protozoology, 36, 24S-27S.

2. Bartlett, M. S., Verbanac, P. A. \& Smith, J. W. 1979. Cultivation of Pneumocystis carinii with WI-38 cells. Journal of Clinical Microbiology, 10, 796-799.

3. Barton, E. G. \& Campbell, W. G. JR. 1969. Pneumocystis carinii in the lungs of rats treated with cortisone acetate. American Journal of Pathology, 54, 209-236.

4. Beveriy, S. M., Ellenderger, T. E., \& Cordingley, J. S. 1986. Primary structure of the gene encoding the bifunctional dihydrofolate reductase-thymidylate synthase of Leishmania major. Proceedings of the National Academy of Sciences, USA, 83, 2584-2588.

5. BZIK, D. J., LI, W.-B., HoRII, T. \& INSELbURG, J. 1987. Molecular cloning and sequence analysis of the Plasmodium falciparum dihydrofolate reductase-thymidylate synthase gene. Proceedings of the National Academy of Sciences, USA, 84, 8360-8364.

6. Chagas, C. 1909. Nova tripanozomiaze humane. Memórias do Instituto Oswaldo Cruz, 1, 159-218.

7. Cushion, M. T. 1989. In vitro studies of Pneumocystis carinii. Journal of Protozoology, 36, 45-52.

8. Cushion, M. T., DeStefano, J. A. \& Walzer, P. D. 1988. Pneumocystis carinii: surface reactive carbohydrates detected by lectin probes. Experimental Parasitology, 67, 137-147.

9. Cushion, M. T. \& Ebrets, D. 1990. Growth and metabolism of Pneumocystis carinii in axenic culture. Journal of Clinical Microbiology, 28, 1385-1394.

10. Cushion, M. T., Stringer, J. R., \& Walzer P. D. 1991. Cellular and molecular biology of Pneumocys. tis carinii. International Reviews in Cytology, 131, 59-107.

11. Cushion, M. T. \& Walzer, P. D. 1984. Growth and serial passage of Pneumocystis carinii in the A549 cell line. Infection and Immunity, 44, 245-251.

12. DeStefano, J. A., Cushion, M. T., Puvanesarajah, V. \& Walzer, P. D. 1990. Analysis of Pneumocystis carinii cyst wall. II. Sugar composition. Journal of Protozoology, 37, 436-441.

13. DeStefano, J. A., Cushion, M. T., Sleight, R. E. \& Walzer, P. D. 1990. Analysis of Pneumocystis carinii cyst wall. I. Evidence for an outer surface membrane. Journal of Protozoology, 37, 428-435.

14. DeStefano, J. A., Cushion, M. T., Trinkle, L. S. \& Walzer, P. D. 1989. Lectins as probes to Pneumocystis carinii surface glycocomplexes. Journal of Protozoology, 36, 65S-66S.

15. Edman, J. C., Edman, U., Cao, M., Lundgren, B., Kovacs, J. A. \& Santi, D. V. 1989. Isolation and expression of the Pneumocystis carinii dihydrofolate reductase gene. Proceedings of the National Academy of Sciences, USA, 86, 8625-8629.

16. Edman, J. C., Kovacs, J. A., Masur, H., Santi, D. V., Elwood, H. J. \& Sogin, M. L. 1989. Ribosomal RNA genes of Pneumocystis carinii. Journal of Protozoology, 36, 18S-20S.

17. Edman, U., Edman, J. C., Lundgren, B. \& Santi, D. V. 1989. Isolation and expression of the Pneumocystis carinii thymidylate synthase gene. Proceedings of the National Academy of Sciences, USA, 86, 6503-6507.

18. Hong, S-T., Steele, P. E., Cushion, M. T., Walzer, P. D., Stringer, S. L. \& Stringer, J. R. 1990. Pneumocystis carinii karyotypes. Journal of Clinical Microbiology, 28, 1785-1795.

19. Kaneshiro, E. S., Cushion, M. T., Walzer, P. D. \& Jayasimhulu, K. 1989. Analyses of Pneumocystis fatty acids. Journal of Protozoology, 36, 69S-72S.

20. Latorre, C. R., Sulzer A. T. \& Norman, L. G. 1977. Serial propagation of Pneumocystis carinii in cell line cultures. Applied and Environmental Microbiology, 33, 1204-1206.

21. Lundgren, B., Cotton, R., Lundgren, J. D., Edman, J. C. \& Kovacs, J. A. 1990. Identification of Pneumocystis carinii chromosomes and mapping of five genes. Infection and Immunity, 58, 1705-1710. 
22. Matsumoto, Y. \& Yoshida, Y. 1984. Sporogony in Pneumocystis carinii: synaptonemal complexes and meiotic nuclear divisions observed in precysts. Journal of Protozoology, 31, 420-428.

23. Murphy, M. J., Pifer, L. L. \& Hughes, W. T. 1977. Pneumocystis carinii in vitro. A study by scanning electron microscopy. American Journal of Pathology, 86, 387-401.

24. Palluault, F., Dei-Cas, E., Slomianny, C., Soulez, B. \& Camus D. 1990. Golgi complex and lysosomes in rabbit derived Pneumocystis carinii. Biology of the Cell, 70, 73-82.

25. Pifer, L. L., Hughes, W. T. \& Murphy, M. J. 1977. Propagation of Pneumocystis carinii in vitro. Pediatric Research, 11, 305-316.

26. Ruffolo, J. J., Cushion. M. T. \& Walzer, P. D. 1989. Ultrastructural observations on life cycle stages of Pneumocystis carinii. Journal of Protozoology, 36, 535-54S.

27. Schmatz, D. M., Romanchek, M. A., Pittarelli, L. A., Schwartz, R. E., Fromtling, R. A., Nollstadt, K. H., Vanmiddlesworth, F. L., Wilson, K. E. \& Turner, M. J. 1990. Treatment of Pneumocystis carinii pneumonia with 1,3 $\beta$-glucan synthesis inhibitors. Proceedings of the National Academy of Sciences, USA, 87, 5950-5954.

28. Stringer, S. L., Hong, S-T., Giuntoli, D. \& Stringer, J. R. 1991. Repeated DNA in Pneumocystis carinii. Journal of Clinical Microbiology, 29, 1194-1201.

29. Stringer, S. L., Hudson, K., Blase, M. A., Walzer, P. D., Cushion, M. T. \& Stringer, J. R. 1989. Sequence from ribosomal RNA of Pneumocystis carinii compared to those of four fungi suggests an ascomycetous affinity. Journal of Protozoology, 36, 14S-16S.

30. Tegoshi, T. \& Yoshida, Y. 1989. New system of in vitro cultivation of Pneumocystis carinii without feeder cells. Journal of Protozoology, 36, 29S-30S.

31. Vavra, J. \& Kucera, K. 1970. Pneumocystis carinii Delanoë, its ultrastructure and ultrastructural affinities. Journal of Protozoology, 17, 463-483.

32. Wakefield, A. E., Pixley, F. J., Banerji, S., Sinclatr, K., Miller, R. F., Moxon, E. R. \& Hopkin J. M. 1990. Amplification of mitochondrial ribosomal RNA sequences from Pneumocystis carinii DNA of rat and human origin. Molecular and Biochemical Parasitology, 43, 69-76.

33. Walzer, P. D., Kim. C. K. \& Cushion, M. T. 1989. Pneumocystis carinii. In: P. D. Walzer \& R. M. Genta (Eds) Parasitic Infections in the Compromised Host, pp. 83-178. Marcel Dekker, New York.

34. Watanabe, J., Hori, H., Tanabe, K. \& Nakamura, Y. 1989. Phylogenetic association of Pneumocystis carinii with the Rhizopoda/Myxomycota/Zygomycota 'group' indicated by comparison of $5 \mathrm{~S}$ ribosomal RNA sequences. Molecular and Biochemical Parasitology, 32, 163-167.

35. Yoganathan, T., Lin, H. \& Buck, G. A. 1989. An electrophoretic karyotype and assignment of ribosomal genes to resolved chromosomes of Pneumocystis carinii. Molecular Microbiology, 3(11), 1473-1480.

36. Yoshikawa, H., Morioka, H. \& Yoshida, Y. 1987. Freeze-fracture localization of filipin-sterol complexes in plasma- and cyto-membranes of Pneumocystis carinii. Journal of Protozoology, 34, 131-137. 